\title{
The Photon Hall Pinwheel Radiation of Angular Momentum by a Diffusing Magneto-optical Medium
}

\author{
B.A. van Tiggelen* \\ Univ. Grenoble Alpes, CNRS, LPMMC, 38000 Grenoble, France \\ G.L.J.A. Rikken \\ Univ. Grenoble Alpes, CNRS, LNCMI, EMFL, Toulouse/Grenoble, France
}

(Dated: May 26, 2020)

\begin{abstract}
We consider an optically thick spherical agglomerate of magneto-optical scatterers with a central isotropic, unpolarized light source, placed in a homogeneous magnetic field. The Photon Hall Effect induces a rotating Poynting vector, both inside and outside the medium. We show that electromagnetic (orbital) angular momentum leaks out and induces a torque proportional to the injection power of the source and the photon Hall angle. This effect represents a novel class of optical phenomena, generating angular momentum from diffusive magneto-transport.
\end{abstract}

Keywords: optical forces, light scattering

\section{INTRODUCTION}

The Photon Hall Effect (PHE) was predicted [1] and measured [2] more than 20 years ago. It refers to the electromagnetic flux that is scattered in a preferential direction perpendicular to both incident current and magnetic field, much in the spirit of the (anomalous) Hall effect in electronic conduction. The PHE has been shown to originate from Faraday rotation in single scattering from a dielectric Mie sphere [3] and vanishes in the regime of pure electric dipole coupling (the Rayleigh regime). Therefore, the PHE does not occur in single scattering of light by atoms, but is in that case generated by multiple scattering [4], or when the electric-dipole transition interferes with a higher multipole [5]. In recent literature many more or less related effects have been identified, such as the photon spin Hall effect [6 8], the quantum spin Hall effect of light 9], the phonon Hall effect [10], the plasmon Hall effect [11] and even other photon Hall effects 12 .

In a scattering medium with a central light source, subject to a homogeneous magnetic field $\mathbf{B}_{0}$ along the $z$-axis, the PHE emerges as a current rotating around the field lines. The Poynting vector associated with the PHE is given by $\mathbf{S}_{\mathrm{PHE}}=D_{H} \widehat{\mathbf{B}}_{0} \times \nabla \rho(\mathbf{r}, t)$, with $\rho(\mathbf{r}, t)$ the electromagnetic energy density and $D_{H}\left(B_{0}\right)$ the Hall diffusion constant, whose sign is determined by sense of the Faraday rotation. The simplest case is to consider a point source $P(\mathbf{r}, t)=P(t) \delta(\mathbf{r})$ injecting the power $P$ into an infinite diffuse medium with diffusion constant $D$. For a single shot of energy $W, P(t)=W \delta(t)$, we can insert the well-known solution of the diffusion equation to obtain,

$$
\mathbf{S}_{\mathrm{PHE}}(r, t)=-\frac{W D_{H} r}{16 \pi^{3 / 2}(D t)^{5 / 2}} \exp \left(-\frac{r^{2}}{4 D t}\right) \widehat{\phi}
$$

\footnotetext{
* Correspondence email address: bart.van-tiggelen@grenoble.cnrs.fr
}

whose maximum propagates outwards as $r \sim \sqrt{D t}$ while decaying in time as $1 /(D t)^{3 / 2}$. The existence of such a rotating electromagnetic current is, on its own already, surprising. Standard textbooks on electromagnetism [13 15] insist on the ambiguity in the interpretation of $\mathbf{S}=c_{0} \mathbf{E} \times \mathbf{H}$ as the energy current density, based on the conservation law $\partial_{t} \rho+\nabla \cdot \mathbf{S}=P$ with the obvious freedom to add the curl of an arbitrary vector field, as is the case for the PHE. In homogeneous, conservative media, the directions of Poynting vector and group velocity coincide and the ambiguity is lifted [16]. In $a b$ sorbing media subject to a magnetic field the addition of a curl turned out to be necessary [17]. In inhomogeneous, conservative media this ambiguity has so far never been addressed. In our simple spherical model with elastic multiple scattering, the circulating light does not, in fine, move around energy, and arguably, there is no ambiguity. Yet this same light carries momentum that can be transferred to matter and is thus observable [18]. Indeed, the interpretation of electromagnetic angular momentum (for $\mu=1$ ), $\mathbf{K}(t)=c_{0}^{-2} \int d^{3} \mathbf{r} \mathbf{r} \times \mathbf{S}$, is not subject to this curl ambiguity, since adding any curl to $\mathbf{S}$ would produce an additional finite angular momentum. Radiative angular momentum in matter is part of another controversy [18] which is of minor importance inside our dilute scattering medium, and of no importance outside. The PHE generates the angular momentum,

$$
\mathbf{K}(t)=-\frac{2 D_{H}}{c_{0}^{2}} \widehat{\mathbf{z}} \int d^{3} \mathbf{r} \rho(\mathbf{r}, t)=-\frac{2 D_{H}}{c_{0}^{2}} \widehat{\mathbf{z}} \int_{0}^{t} d t^{\prime} P\left(t^{\prime}\right)
$$

proportional to the total amount of energy injected, and conserved in time after the injection stops. The total torque on the diffuse medium is $\mathbf{N}=-d \mathbf{K} / d t=$ $2 D_{H} \widehat{\mathbf{z}} P(t) / c_{0}^{2}$. This torque is of course very small. For a PHE angle of $D_{H} / D \sim 10^{-3}$, the largest we have realized, a mean free path of $10 \mu \mathrm{m}\left(D \approx c_{0} \ell / 3=1000\right.$ $\mathrm{m} / \mathrm{s}^{2}$ ) and a power of $10 \mathrm{~W}$, we find a torque $N=10^{-16}$ $\mathrm{Nm}$. The conservation of total angular momentum can 


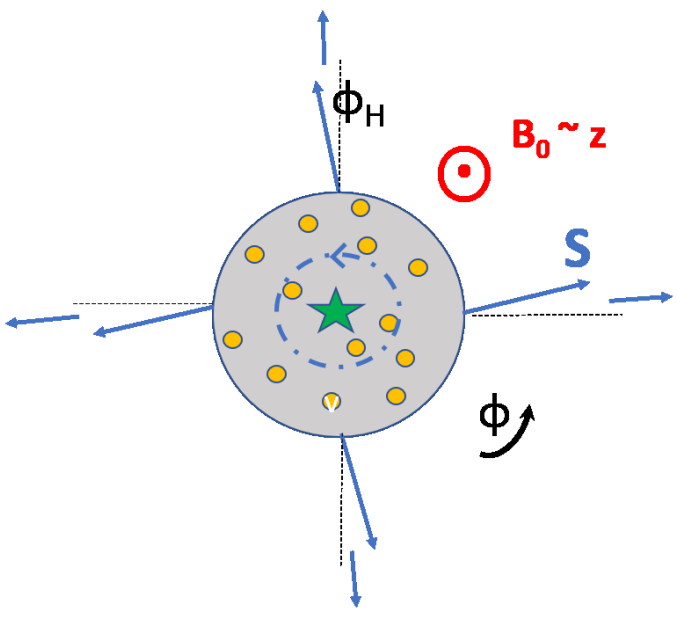

Figure 1. The Photon Hall Pinwheel: The Photon Hall Effect inside a diffuse sphere emerges as a Poynting vector circulating around the origin, not affecting energy transport but carrying a finite orbital angular momentum. The radiation leaves the boundaries under the Photon Hall angle $\phi_{H}=D_{H} / D$ with the local normal (here drawn for $D_{H}<0$ ). This angle decreases as $1 / r$ with distance.

be expressed as

$$
\frac{d}{d t}\left(\mathbf{K}+\mathbf{K}_{\text {mat }}\right)_{i}=\lim _{r \rightarrow \infty} \int d^{2} A(r) \hat{r}_{n} \epsilon_{i j k} \hat{r}_{j} T_{n k}(\mathbf{r}, t)
$$

with $\mathbf{K}_{\text {mat }}$ the angular momentum of the matter in the sphere and $\mathbf{T}$ the symmetrical electromagnetic stress tensor [13]. The term on the righthand side describes the flow of angular momentum to infinity. In typical scattering problems the far field is characterized by $T_{i j}(\mathbf{r})=P \delta_{i j}+Q \hat{r}_{i} \hat{r}_{j}$, so that this flow vanishes trivially. In the presence of the PHE however, it does not. Nevertheless, if we choose $r>c_{0} t$, the leak of angular momentum is outside the light cone and vanishes at infinity. As a result, $\mathbf{K}+\mathbf{K}_{\text {mat }}$ must be conserved in time. They can exchange mutually via the Abraham torque $\mathbf{N}_{A}=c_{0}^{-1} \int d^{3} \mathbf{r} \mathbf{r} \times \partial_{t}(\mathbf{P} \times \mathbf{B})[19]$.

In order to calculate the photon Hall torque on a scattering agglomerate, we consider a finite sphere with radius $a$ (see Figure 1). Inside the sphere the diffusion equation applies as before, and for times $t<a^{2} / 4 D$ after the source emitted the situation is essentially the one of an infinite medium. At later times we have to know how photons leave the sample. In an intuitive approximation, we can imagine the PHE at the boundaries making the photons leave the sphere at the "Photon Hall Angle" $\phi_{H}=D_{H} / D$ with the local normal. If $P(a, t)=4 \pi a^{2} S(a, t)$ is the total current leaving the sphere, the rate of angular momentum that flows outside is $a \times \phi_{H} P(a, t) / c_{0}$. The torque can thus be estimated as $N(t) \approx \phi_{H} P(a, t) a / c_{0}$. Energy conservation imposes that $P(a, t)=P(t)-\partial_{t} W$, with $W$ the electromagnetic energy inside the sphere. If we assume the latter is sta- tionary, we obtain,

$$
N(t)=\frac{D_{H}}{D} \frac{a}{c_{0}} P(t)
$$

To get quantitative insight how angular momentum is radiated by the sphere into space we will follow the standard method to deal with boundaries of the diffusion equation, and calculate the electromagnetic angular momentum outside the sphere, rather than the flow of angular momentum. A good treatment of the skin layer [20] is essential to respect flux conservation. The magneto-optics of the effective medium is described by a complex index of refraction $\varepsilon_{\sigma}(\mathbf{k})$ in terms of which wave vector $k_{\sigma}$ and extinction length $\ell_{\sigma}$ are defined as $\varepsilon_{\sigma}(\mathbf{k}) \omega^{2} / c_{0}^{2}=\left(k_{\sigma}+i / 2 \ell_{\sigma}\right)^{2}$ that here depend both on circular polarization and wave vector direction. In radiative transport, the electric field in and outside the medium can be imagined to be emitted by a diffuse random secondary source as $E_{i}(\mathbf{r})=\int d^{3} \mathbf{r}_{s} G_{i j}\left(\mathbf{r}, \mathbf{r}_{s}\right) s_{j}\left(\mathbf{r}_{s}\right)$. Consequently, the "ensemble-averaged" field correlation function is

$$
\begin{aligned}
\left\langle E_{i}(\mathbf{r}) \bar{E}_{j}\left(\mathbf{r}^{\prime}\right)\right\rangle & =\int d^{3} \mathbf{r}_{S} \int d^{3} \mathbf{x}\left\langle G_{i k}\left(\mathbf{r}, \mathbf{r}_{s}^{+}\right)\right\rangle\left\langle\bar{G}_{l j}\left(\mathbf{r}^{\prime}, \mathbf{r}_{s}^{-}\right)\right\rangle \\
& \times\left\langle s_{k}\left(\mathbf{r}_{s}^{+}\right) \bar{s}_{l}\left(\mathbf{r}_{s}^{-}\right)\right\rangle
\end{aligned}
$$

with $\mathbf{r}_{s}^{ \pm}=\mathbf{r}_{s} \pm \mathbf{x} / 2$. In the far field outside the sphere $G_{i j}\left(\mathbf{r}^{ \pm}\right) \rightarrow\left(\delta_{i j}-\hat{r}_{i} \hat{r}_{j}\right) \exp (i K r) \exp (i k \hat{\mathbf{r}} \cdot \mathbf{x} / 2) /(-4 \pi r)$, and this expression simplies to a manifestation of Huygens' principle,

$$
\left\langle E_{\sigma}(\mathbf{r}) \bar{E}_{\sigma^{\prime}}(\mathbf{r})\right\rangle=\frac{1}{(4 \pi)^{2}} \int d^{3} \mathbf{r}_{s} \frac{e^{-i\left(K_{\sigma}-\bar{K}_{\sigma^{\prime}}\right) b}}{\left|\mathbf{r}-\mathbf{r}_{s}\right|^{2}} S_{\sigma \sigma^{\prime}}\left(\mathbf{k}, \mathbf{r}_{s}\right)
$$

with $b$ the length traversed by the light from the source to its exit point (Figure 2). Because $K_{\sigma}$ is complex-valued inside the sphere, the integral is restricted to the skin layer and the far field correlation function is proportional to the Fourier transform $S\left(\mathbf{k}, \mathbf{r}_{s}\right)$ of the source correlation function $\left\langle s\left(\mathbf{r}_{s}^{+}\right) \bar{s}\left(\mathbf{r}_{s}^{-}\right)\right\rangle$(in radiative transfer proportional to the local specific intensity) with $\mathbf{x}$, integrated over the surface of the sphere. A fundamental result from transport theory, is that in the diffuse regime,

$$
S_{\sigma \sigma^{\prime}}\left(\mathbf{k}, \mathbf{r}_{s}\right)=2 \pi k \delta_{\sigma \sigma^{\prime}} \operatorname{Im} \varepsilon_{\sigma}(\mathbf{k})\left(1-\frac{3}{c_{0}} \hat{\mathbf{k}} \cdot \mathbf{D} \cdot \nabla\right) \rho\left(\mathbf{r}_{s}\right)
$$

This is basically a manifestation of the fluctuation dissipation theorem, with the first term standing for detailed balance, and the gradient term causing the diffuse energy flow. This source function depends on the magneto-optical birefringence of the effective medium, as well as on the PHE via $D_{i j}=D \delta_{i j}+D_{H} \epsilon_{i j z}$. In the far field outside the medium the Poynting vector is just $\mathbf{S}=c_{0} \hat{\mathbf{k}}\left(\hat{\mathbf{r}}_{s}\right)|E(\mathbf{r})|^{2}$, with $\hat{\mathbf{k}}$ the unit vector along the 


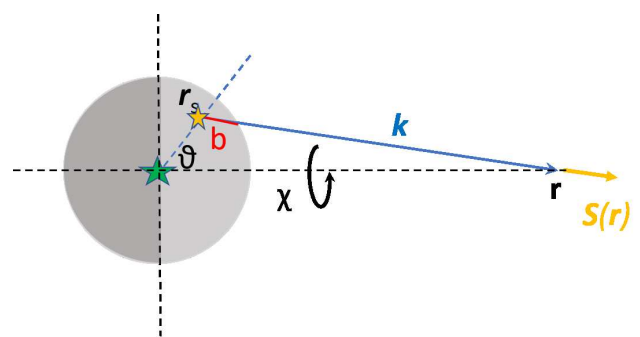

Figure 2. Geometry of the PHE in the far field of the sphere. The diffuse light arriving from the source in the center initiates secondary random sources at $\mathbf{r}_{s}$ within a skin layer of depth $\ell$ near the surface, that contribute to the Poynting vector $\mathbf{S}$ in the far field. The induced PHE at $\mathbf{r}$ is largest when the magnetic field is perpendicular to the plane.

vector $\mathbf{r}-\mathbf{r}_{s}$. We thus find

$$
\begin{aligned}
\mathbf{S}\left(\mathbf{r}, t+\frac{r}{c_{0}}\right) & =\frac{c_{0}}{8 \pi} \sum_{\sigma} \int d^{2} \hat{\mathbf{r}}_{s} \int_{0}^{\infty} d z e^{-z / \mu \ell_{\sigma}} \frac{\hat{\mathbf{k}}\left(\mathbf{r}_{s}\right)}{\left|\mathbf{r}-\mathbf{r}_{s}\right|^{2}} \\
& \times \frac{1}{\ell_{\sigma}}\left(1-\frac{3}{c_{0}} \hat{\mathbf{k}}\left(\mathbf{r}_{s}\right) \cdot \mathbf{D} \cdot \nabla\right) \rho\left(a \hat{\mathbf{r}}_{s}, t\right)
\end{aligned}
$$

with $\mu=\cos \theta, b \approx z / \mu$ and the integral of $\mathbf{r}_{S}$ extending over half the outer surface of the sphere visible from $\mathbf{r}$. Hence,

$$
\begin{array}{r}
\quad \mathbf{S}\left(\mathbf{r}, t+\frac{r}{c_{0}}\right)=\frac{c_{0}}{4 \pi} \int d^{2} \hat{\mathbf{r}}_{s} \frac{\mu \hat{\mathbf{k}}\left(\mathbf{r}_{s}\right)}{\left|\mathbf{r}-\mathbf{r}_{s}\right|^{2}} \\
\times\left(\rho(a, t)-\frac{3}{c_{0}} \hat{\mathbf{k}}\left(\mathbf{r}_{s}\right) \cdot \mathbf{D} \cdot \hat{\mathbf{r}}_{s} \partial_{r} \rho(a, t)\right)
\end{array}
$$

The only magneto-optical effect that has survived in this expression for the Poynting vector is the PHE contained in $\mathbf{D}$. The leading radial flow of energy is obtained by putting $\hat{\mathbf{k}} \approx \hat{\mathbf{r}}$, and becomes

$$
\begin{aligned}
\mathbf{S}_{r}\left(\mathbf{r}, t+\frac{r}{c_{0}}\right) & =\frac{c_{0} a^{2}}{2 r^{2}} \hat{\mathbf{r}} \int_{0}^{1} d \mu \mu\left(\rho(a, t)-\frac{3}{c_{0}} \mu D \partial_{r} \rho(a, t)\right) \\
& =\frac{c_{0} a^{2}}{4 r^{2}} \hat{\mathbf{r}}\left(\rho(a, t)-\frac{2}{c_{0}} D \partial_{r} \rho(a, t)\right)
\end{aligned}
$$

The usual radiative boundary condition at the surface is $\rho+\frac{2}{c_{0}} D \partial_{r} \rho=0$, and identifies $z_{0}=2 D / c_{0}$ as the extrapolation length [20]. The total current leaving the sphere at distance $r$ is therefore $J(r, t)=4 \pi r^{2} S_{r}=$ $-4 \pi a^{2} D \partial_{r} \rho\left(a, t-r / c_{0}\right)$. The PHE vanishes in the same approximation $\hat{\mathbf{k}} \approx \hat{\mathbf{r}}$ since for any fixed angle $\theta$, the azimuthal integral $\int d \chi \mathbf{r}_{s}$ (Fig. 2) is directed along $\mathbf{r}$, and $\hat{\mathbf{r}} \cdot \mathbf{D}_{H} \cdot \hat{\mathbf{r}}=0$. Since $\hat{\mathbf{k}}=\hat{\mathbf{r}}\left(1+\mu r_{s} / r\right)-\mathbf{r}_{S} / r+\mathcal{O}\left(1 / r^{2}\right)$ the $\mathrm{PHE}$ generates a Poynting vector in the far field given by

$$
\begin{aligned}
\mathbf{S}(\mathbf{r}, t)= & \frac{c_{0} a^{2}}{4 \pi r^{2}} \int_{0}^{1} d \mu \int_{0}^{2 \pi} d \chi \mu\left(\frac{-\mathbf{r}_{S}}{r}\right) \hat{\mathbf{r}} \cdot\left(\hat{\mathbf{r}}_{s} \times \hat{\mathbf{z}}\right) \\
& \times \frac{-3 D_{H}}{c_{0}} \partial_{r} \rho\left(a, t-\frac{r}{c_{0}}\right) \\
= & \frac{3}{16} \frac{a^{2}}{r^{3}} D_{H} \partial_{r} \rho\left(a, t-\frac{r}{c_{0}}\right)(\hat{\mathbf{z}} \times \hat{\mathbf{r}})
\end{aligned}
$$

This expression corresponds to an Hall angle outside the sphere equal to $S_{\phi} / S_{r}=(3 / 16)\left(D_{H} / D\right) a / r$ with the same sign as inside the sphere, that decays slowly with distance. The angular momentum carried by the leaking electromagnetic field is

$$
\begin{aligned}
\mathbf{K} & =\frac{1}{c_{0}^{2}} \int d^{3} \mathbf{r} \mathbf{r} \times \mathbf{S} \\
& =-\hat{\mathbf{z}} \frac{D_{H}}{D} \frac{a}{8 c_{0}^{2}} \int_{a}^{\infty} d r J\left(a, t-\frac{r}{c_{0}}\right)
\end{aligned}
$$

in terms of the total energy current $J(a, t)$ leaving the sphere. It is directed along the vector $-D_{H} \mathbf{B}_{0}$ and propagates outwardly. This conclusion can also be obtained by considering the righthand side of Eq. (3). The total angular momentum grows with time as longs as energy is injected into the sphere. Since $J(t<0)=0$ the radial integral extends until $r=c_{0} t$ so that we can identify the torque on the sphere as

$$
\mathbf{N}=-\frac{d \mathbf{K}}{d t}=\hat{\mathbf{z}} \frac{D_{H}}{D} \frac{a}{8 c_{0}} J\left(a, t-\frac{a}{c_{0}}\right)
$$

Equation (13) is valid for $a \gg \ell$. It is basically equivalent to the result obtained in Eq.(4). The extra factor $1 / 8$ stems from the fact that not all photons leave the diffuse medium at the photon Hall angle. For a photon Hall angle of $10^{-3}, a=1 \mathrm{~mm}$ and $P=10 \mathrm{~W}$, this yields $N=4 \cdot 10^{-15} \mathrm{Nm}$. The observation of such a value is within experimental reach using a torsion pendulum [21]. Figure 3 shows a possible implementation of an experiment to observe this effect.

Under constant flux during a time $T$, the total angular momentum irreversibly radiated into space is much bigger than the angular momentum $K \sim D_{H} W_{S} / c_{0}^{2}$ derived in Eq. (2) reversibly taken from the sphere during radiation. With a moment of inertia $I=(8 \pi / 15) \rho_{m} a^{5}$ the angular velocity of the sphere grows in time as $\varpi=N \times T / I$. After an exposure time of one hour, and a mass density $\rho_{M}=1,5 \mathrm{~g} / \mathrm{cm}^{3}$ (corresponding to glycerol doped with a $10 \%$ volume fraction $\mathrm{EuF}_{2}$ particles at $4,2 \mathrm{~K} ; \mathrm{EuF}_{2}$ has a large Faraday rotation of $1000 \mathrm{rad} / \mathrm{m}$ for a magnetic field of 1 Tesla), we find $\varpi=10 \mathrm{rad} / \mathrm{s}$. This is not small at all. The photon Hall pinwheel effect is not small either from the perspective of a single photon. Equation (12) tells that the total angular momentum is proportional to the product of $\phi_{H}\left(a / c_{0}\right)$ and the total electromagnetic energy $W$ radiated. If we write $W=n \hbar \omega$ with $n$ the number of injected photons, the total angular momentum carried away per photon, and measured in units of $\hbar$, is given by $K / n \hbar=\phi_{H} k a / 8$. A sphere of $1 \mathrm{~mm}$ in radius has $k a=12000$ at optical frequencies. For $\phi_{H} \approx 10^{-3}$, each photon leaving the sphere takes away roughly one $\hbar$ of angular momentum.

The photon Hall pinwheel effect outlined above represents an entire novel class of phenomena, that link diffusive transport in a magnetic field to angular momentum. Other diffusive magneto-transport effects, like the 


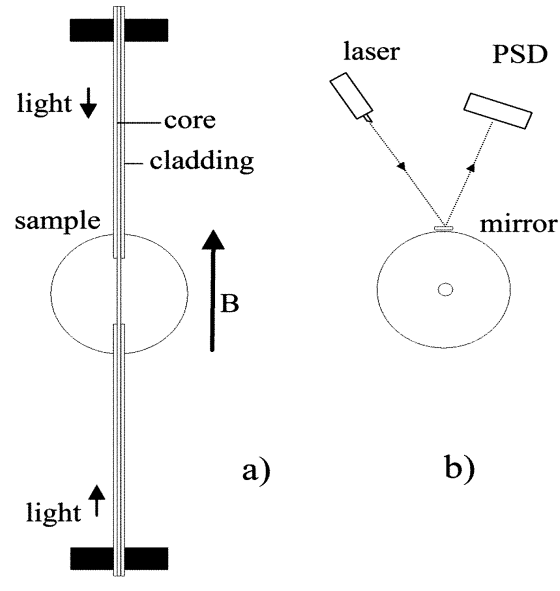

Figure 3. Proposed experimental setup for the observation of the photon Hall pinwheel effect (not to scale). a) side view. The optical fiber serves as torsion wire, with the fiber cladding removed near the center of the spherical agglomerate of scatterers (sample) with radius a. The light injected into the two fiber ends is chopped at the torsion pendulum resonance frequency. b) Mid-plane top view. Measurement of the torsion angle by deflection of a laser onto a position sensitive detector, which is read out with a phase sensitive detector at the light chopping frequency ones mentioned in the introduction, should lead to similar pinwheel effects. An analogy can be argued to exist between this effect and the Einstein-De Haas effect, that establishes the link between magnetism and angular momentum. In particular, we conjecture that a star, in possession of a strong magnetic field, might be subject to a torque. Low energy free electrons are often a major source of radiative transfer [22] via multiple Thomson scattering, which is affected by the magnetic field [23].

In summary, we have demonstrated the existence of a circularly rotating Poynting vector, induced by the photon Hall effect in a sphere consisting of random magnetooptical scatterers, illuminated by a central optical source, and placed in a homogenous magnetic field. This Halltype photon flux carries a finite orbital angular momentum. Surprisingly, the radiation leaving the sphere carries angular momentum with it, and exerts a torque on the sphere. Realistic estimates show that the observation of this effect is within experimental reach.

We thank Robert Whitney for drawing attention to the pinwheel effect.
[1] B.A. van Tiggelen, Phys. Rev. Lett. 75, 422 (1995); D. Lacoste and B. A. van Tiggelen EPL 45, 721- 725 (1999).

[2] G.L.J.A. Rikken and B.A. van Tiggelen, Nature 381, 54 (1996); G. Duechs, A. Sparenberg, G.L.J.A. Rikken, B. A. van Tiggelen Phys. Rev. E 62, 2840 (2000).

[3] D. Lacoste, B.A. van Tiggelen, G.L.J.A. Rikken, A. Sparenberg JOSA A15, 1636-1642 (1998).

[4] B. Gremaud, D. Delande, O. Sigwarth and Ch. Miniatura, Phys. Rev. Lett. 102,217401 (2009).

[5] B.A. van Tiggelen and D. Wilkowski, Eur. Phys. J. (Special Topics) 226 (7), 1515-1523 (2017).

[6] A. Kavokin, G. Malpuech, and M. Glazov Phys. Rev. Lett. 95, 136601 (2005).

[7] T. Bardon-Brun, D. Delande and N. Cherroret, Phys. Rev. Lett. 123, 043901 (2019).

[8] X. Ling, X. Zhou, K. Huang, Y. Liu, C. Qiu, H. Luo, and S. Wen, Rep. Prog. Phys. 80, 066401 (2017).

[9] K. Y. Bliokh, D. Smirnova, F. Nori, Science 348 (6242), 1448-1451 (2015).

[10] C. Strohm, G. L. J. A. Rikken, and P. Wyder Phys. Rev. Lett. 95, 155901 (2005).

[11] G. Duechs, G. L. J. A. Rikken, T. Grenet, and P. Wyder Phys. Rev. Lett. 87, 127402 (2001).

[12] D. Jahani, A. Alidoust Ghatar, L. Abaspour, and T. Jahani, J. App. Phys. 124, 043104 (2018).

[13] J.D. Jackson, Classical Electrodynamics (John Wiley, 1975); see page 237 for a discussion on the uniqueness of Poynting vector.

[14] M. Born and E. Wolf, Optics (Pergamon, 1980), sixth edition; see section 1.1.4 for a discussion on the arbitrariness of the Poynting vector.

[15] R. P. Feynman, R. Leighton and M. Sands, The Feyn- man Lectures on Physics: Electromagnetism and Matter, (Addison-Wesley, 1964), see section 27-4 for a discussion on the arbitrariness of electromagnetic energy flow.

[16] L.D. Landau, E.M. Lifshitz and L.P. Piteavskii, Electrodynamics of Continuous Media, Vol 8 (Pergamon, 1984), paragraph 97.

[17] G.L.J.A. Rikken and B.A. van Tiggelen, Phys. Rev. Lett. 78, 847 (1997)

[18] M. Kristensen and J.P. Woerdman, Phys. Rev. Lett. 72, 2171 (1994).

[19] B. A. van Tiggelen Phys. Rev. A 100, 053826 (2019).

[20] Th. M. Nieuwenhuizen and J. M. Luck Phys. Rev. E 48, 569 (1993).

[21] G. T. Gillies and R. C. Ritter, Rev. Sci. Instrum. 64, 283 (1993).

[22] G.B. Rybicki and A. P. Lightman, Radiative Processes in Astrophysics (Wiley, 1979).

[23] T. Hamada, and S. Kanno, Publ. Astr. Soc. Japan, 26(4), 421-427 (1974). 